\title{
DEM-BASED STUDY OF HABITAT CHARACTERISTICS OF TORREYA FENGQIAO IN KUAIJI MOUNTAIN, CHINA
}

\author{
Xiaoming Wang ${ }^{1,2}$, Ke Wang $^{1, *}$, Weijiu Ao ${ }^{1}$, Jinsong Deng ${ }^{1}$ \\ ${ }^{1}$ Institution of Remote Sensing \& Information Technology Application, Zhejiang University, \\ Hangzhou, Zhejiang Province, P. R. China 310029 \\ ${ }^{2}$ Research Institute of Subtropical Forestry, China Academy of Forestry, Hangzhou, Zhejiang \\ Province, P. R. China 310029 \\ * Corresponding author, Address: Institution of Remote Sensing \& Information Technology \\ Application, Zhejiang University, Hangzhou, Zhejiang Province, P. R. China, Tel: +86- \\ 571-86971272, Fax: +86-571-86971272,Email: kwang@zju.edu.cn
}

\begin{abstract}
The habitat characteristics of Torreya were investigated based on high resolution DEM and remote sensing image. The distribution of Torreya was mapped by visual interpretation of IKONOS image. The landform and PCA analysis results showed that curvature, altitude, aspect and slope were found to be the dominant features controlling spatial distribution pattern of Torreya. A certain degree moisture related variables were proved to be the most important parameters to explain Torreya habitats preferences. The preferable habitat requirement for Torreya focused on those concave and southwest hillsides with elevation between 400 and $600 \mathrm{~m}$ and slope less than $30^{\circ}$.
\end{abstract}

Keywords: vegetation distribution, digital elevation model, landform analysis, GIS

\section{INTRODUCTION}

Accurately understanding the native habitat for rare plants with restricted geographic distributions is an important component of plant conservation and development planning. Remotely sensed data have been widely used for assisting in vegetation mapping in the last few years and have been proved an effective tool. They offer the possibility of extrapolating mapping results,

Please use the following format when citing this chapter:

Wang, X., Wang, K., Ao, W. and Deng, J., 2009, in IFIP International Federation for Information Processing, Volume 293, Computer and Computing Technologies in Agriculture II, Volume 1, eds. D. Li, Z. Chunjiang, (Boston: Springer), pp. 161-168. 
especially in large and hardly accessible remote areas (Kalliola and Syrjänen, 1991). Meantime, topographical attributes derived from Digital Elevation Model (DEM) are widely used in mountain ecology (Moore et al. 1991; Hoersch et al., 2002; Garcia et al., 2007), offering a much more reliable predictor database compared with direct climatic or edaphic site factors (Davis and Goetz, 1990). These works have served to the understanding of the complex interactions between spatial distribution patterns of site factors and vegetation types. However, compared to common species, the endemic and rare plants with a narrow size distribution showed quite different in habitat selection. It is challenging, due to lack of information synthesis on the ecology of rare plants, lack of spatial data for relevant environmental attributes and scales. And there have been few studies of analysing preference habitat for rare species based on the level of species (Claessens et al., 2006, Tatsuhara and Antatsu, 2007). Fortunately, with the recent development of high resolution IKONOS or QUICKBIRD remote sensing images and DEMs, it provides a new opportunity to make a thorough analysis to the site selection and distribution patterns of rare species.

Torreya Fengqiao (Torreya grandis var. Merrillii), an evergreen conifer in genus Torreya of family Taxaceae, is famous for its precious rare nut in China (Li Zhangju et al., 2005). Researches relating to its distribution and habitat requirements have been carried out for the last decades including biological characteristics (Ren Qinliang, 1989), suitability of introduction and cultivation (Liu Quan et al., 1993), climatic requirements (Fu Yulu et al., 1999) and spatial distribution (Meng Hongfei et al., 2003). However, our knowledge about interactions of its distribution and environmental factors is rather poor (Han Ninglin et al., 2006). In the present study, a combination of DEM, remotely sensed image and field data were used to find a statistical correlation with the spatial distribution pattern of Torreya Fengqiao. The major objective of this study was to find the habitat characteristics of Torreya Fengqiao in Kuaiji Mountains for its sustainable development.

\section{STUDY AREA AND METHOD}

\section{$2.1 \quad$ Study area}

The study area, Kuaiji Mountains, is located at northeastern Zhejiang province, East China (Fig. 1). The orientation of mountain is from southwest to northeast. Its length is $90 \mathrm{~km}$ from south to north and the width was $30 \mathrm{~km}$ from east to west. The core zone of Torreya Fengqiao is located in Zhaojia town, Zhuji County $\left(121^{\circ} 29^{\prime}-121^{\circ} 33^{\prime} \mathrm{E}, 29^{\circ} 40^{\prime}-29^{\circ} 44^{\prime} \mathrm{N}\right)$, lying in the 
middle part of the Kuaiji Mountains and covering an area of $27 \mathrm{~km}^{2}$, Elevation ranges from 90 to $830 \mathrm{~m}$ above sea level. The climate is characterized as subtropical monsoon zone. More than 28,000 ancient trees (about 200 to 600 years old), with trunks up to 2.0 meters in diameter and heights up to 15 meters, still survive in the wild in this area (Meng Hongfei et al., 2003).

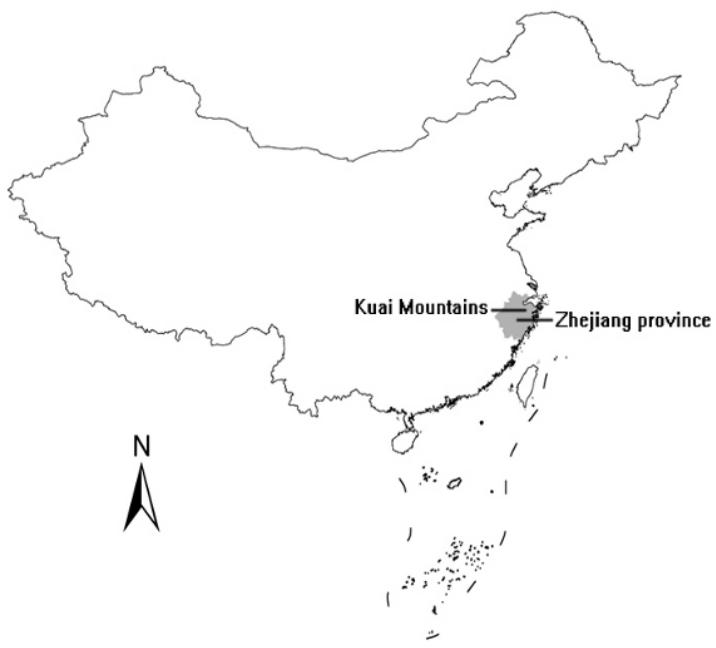

Fig. 1. The location of Kuaiji Mountains in Zhejiang province, China.

\subsection{Data and data preparation}

\subsubsection{DEM data}

The DEM data was derived from the topographic maps at the scale of 1:10,000 using the software of ArcGIS 9.2. The contours were firstly digitized, then the linear map was used to build TIN, and at last, the DEM with $10 \mathrm{~m}$ resolution was produced. Several topographical variables such as elevation, slope, aspect, curvature, hillshade and topographically derived variables (wetness index, sinaspect and cosaspect) were used as possible environment related explanatory variables to describe habitat of Torreya Fengqiao. The respective variable grid layer was computed from the 10-m spatial resolution DEM and ArcGIS software. For more details about the landform parameters see Hoersch et al. (2002). 


\subsubsection{Remotely sensed data}

IKONOS image was fused using an PANSHARP transformation, then orthorectified based on a 1:10,000 digital topographic map, a Digital Elevation Model and PCI Orthobase software, resulting in fused image with spatial resolution of $1 \mathrm{~m}$ (PCI Geomatics, 1998). Considering the complicated features of forest cover in study area, different ways were tried to improve the accuracy of classification, and visual interpretation was finally used for Torreya mapping. An example of recognition for Torreya Fengqiao trees extraction was showed in Fig. 2. A total of 215 patches of Torreya were identified.. Torreya trees were classified rather well and the classification accuracy based on 56 validation points amounted to $89.6 \%$ with $8 \%$ standard deviation. Inaccuracies were caused by those scattered Torreya trees with little crown among other type of dense forests.

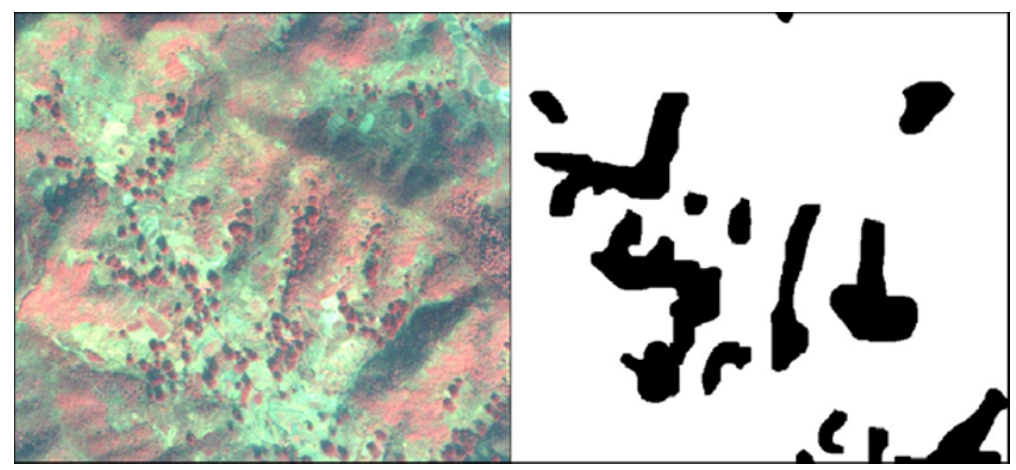

Fig.2. Pattern recognition for Torreya extraction: IKONOS image (left) and resulting (right)

\subsection{Methods}

Firstly, Torreya distribution patches and ten grid layers of environmental variables was generated based on IKONOS image and DEM data. Then the analysis of correlation between the spatial distribution of Torreya Fengqiao and its site characteristics with the grade data of environmental variables was performed in GIS for Finally, quantitative analysis was carried out with the help of Social Professional Statistics Software (SPSS) for Principal Components Analysis (Hoersch et al., 2002). 


\section{RESULTS AND DISCUSSION}

\subsection{Relationship between topography and spatial distribution of Torreya}

The relationship between spatial distribution of Torreya Fengqiao and the characteristics of landform are described in Table 1. Topographical parameters such as curvature, elevation, slope, and aspect seems having high correlation with its distribution and they appear to be very useful in explaining the occurrence, distribution and abundance of Torreya Fengqiao. It showed that the preferable habitat requirement for Torreya Fengqiao focused on those concave and southwest areas with elevation between 400 and $600 \mathrm{~m}$ and slope less than $30^{\circ}$.

Table 1. Habitat characteristics of Torreya Fengqiao in Kuaiji Mountains

\begin{tabular}{|c|c|c|c|}
\hline Variable & Classes & Percent & Description \\
\hline \multirow{3}{*}{ curvature } & $>0$ & 36.13 & Convex shape \\
\hline & $=0$ & 0.72 & Flat \\
\hline & $<0$ & 63.15 & Concave shape \\
\hline \multirow{3}{*}{ DEM } & 1 & 20.49 & $<400 \mathrm{~m}$ \\
\hline & 2 & 76.14 & $400-600 \mathrm{~m}$ \\
\hline & 3 & 3.36 & $>600 \mathrm{~m}$ \\
\hline \multirow{5}{*}{ aspect } & 1 & 22.64 & $0-90^{\circ}$ \\
\hline & 2 & 24.18 & $90-180^{\circ}$ \\
\hline & 3 & 32.51 & $180-270^{\circ}$ \\
\hline & 4 & 20.49 & $270-360^{\circ}$ \\
\hline & 5 & 0.17 & Flat \\
\hline \multirow{3}{*}{ sinaspect } & $<0$ & 53.17 & West slope \\
\hline & $=0$ & 0.02 & Flat \\
\hline & $>0$ & 46.82 & East slope \\
\hline \multirow{3}{*}{ cosaspect } & $>0$ & 43.31 & North slope \\
\hline & $=0$ & 0.01 & Flat \\
\hline & $<0$ & 56.68 & South slope \\
\hline \multirow{4}{*}{ slope } & 1 & 2.80 & $<10^{\circ}$ \\
\hline & 2 & 20.52 & $10-20^{\circ}$ \\
\hline & 3 & 43.98 & $20-30^{\circ}$ \\
\hline & 4 & 32.70 & $>30^{\circ}$ \\
\hline
\end{tabular}

According to fieldwork, different elevation region in Kuaiji Mountains showed important difference in spatial pattern due to their various site factors. In lower elevation areas, it only located along the narrow streams and adjacent slopes which could afford a moist and cool habitat. So, only a little proportion $(20.49 \%)$ survived in this zone. However, $76.14 \%$ of it was 
located between 400-600 $\mathrm{m}$ in elevation. Mature trees in this area are thriving in open, sunny and exposed locations. It seems to be a relatively dry microsite, but more than $60 \%$ are on locally concave topography and mid to lower slopes in order to keep with steady soil moisture. Elevation condition is recognized as an important mechanism for its occurrence, because it has been able to altitudinally compensate for rises in temperature during summer dryness.

\subsection{Principal components analysis}

Results of the PCA using the whole data set are shown in Table 2. About 5 principal components explain over $80 \%$ of the 10 landform features. The first component represented curvature relating somehow to a certain soil moisture requirement. The second is a combination of aspect and hillshade representing solar radiation and soil moisture. The third component was highly correlated with elevation. This partly supported the results of topography analysis (Table. 1) which meant Torreya species exhibited high selectivity of elevation belt. And the last two components stressed the meaning of slope and aspect for Torreya which also had great correlation with soil moisture and solar radiation. Therefore, it could be concluded that a certain range of curvature on distinct aspects and altitudinal belt are important for Torreya. Since most of major influencing factors had strong correlation with soil moisture, it suggested that a certain degree moisture related variables proved to be important parameters to explain Torreya habitats preferences.

Table 2. Cumulative eigenvaules and Eigenmatrix for the overall Principal Component Analysis (PCA)

\begin{tabular}{cccccccc}
\hline \multirow{2}{*}{ PCA } & Cumulative variance & Parameter & \multicolumn{5}{c}{ Component } \\
\cline { 4 - 8 } & & & 1 & 2 & 3 & 4 & 5 \\
\hline 1 & $\mathbf{2 5 . 1 4}$ & DEM & .366 & -.007 & $\mathbf{. 9 0 5}$ & -.171 & .003 \\
2 & $\mathbf{4 6 . 5 7}$ & aspect & .046 & $\mathbf{. 8 0 5}$ & -.084 & -.347 & -.279 \\
3 & $\mathbf{6 4 . 0 0}$ & sinaspect & -.018 & -.883 & .054 & .234 & .272 \\
4 & $\mathbf{7 8 . 3 6}$ & cosaspect & -.065 & .358 & .163 & $\mathbf{. 6 3 0}$ & $\mathbf{. 6 4 3}$ \\
5 & $\mathbf{8 8 . 6 3}$ & slope & .188 & -.005 & -.295 & -.660 & .500 \\
6 & 93.845 & wetness & -.581 & -.011 & .167 & .496 & -.435 \\
7 & 97.02 & curvature & $\mathbf{. 9 2 8}$ & .000 & -.211 & .270 & -.128 \\
8 & 99.27 & plancurve & $\mathbf{. 8 0 1}$ & .000 & -.254 & .167 & -.004 \\
9 & 99.71 & procurve & $\mathbf{- . 7 8 6}$ & .000 & .114 & -.286 & .203 \\
10 & 100 & hillshade & -.033 & $\mathbf{. 8 9 6}$ & .078 & .289 & .259 \\
\hline
\end{tabular}

For the biological characteristics of Torreya Fengqiao, several important periods during growth and development stage such as miosis of floral organ, anthesis, pollination and fruit ripeness have many affinities with moisture condition. The shallow roots of the species further exacerbate its 
vulnerability to water deficits especially in the period of high temperature and dryness in summer (Ren Qinliang, 1984; Wu Jungen et al., 1994; Han Ninglin et al., 2006). The growth patterns and site-selectivity were consistent with the moisture preferences of Torreya species.

\section{CONCLUSION}

Environmental factors have been considered as the main determinant in vegetation distribution (Leduc et al., 1992). Torreya Fengqiao has presumably survived from countless natural disaster in its native habitat for millennia; this suggests that the current spatial distribution pattern is most suitable for its growth. Based on high resolution DEM and IKONOS image, some basic rules of spatial distribution of Torreya Fengqiao are proposed. Curvature, altitude, aspect and slope were found to be the dominant features controlling spatial pattern of Torreya Fengqiao in study area. The study concludes that the occurrence of Torreya Fengqiao in this region largely concentrates on those concave and southwest hillsides with slope lower than $30^{\circ}$ and elevation belt between 400 and $600 \mathrm{~m}$. The results suggest that a certain degree moisture related variables proved to be the most important parameters to explain its habitats preferences. Understanding relationships between environmental variables and spatial distribution of Torreya Fengqiao in this area helps us to apply these findings in management, reclamation, and development of Torreya plantation.

\section{ACKNOWLEDGEMENTS}

Financial supports from China National Natural Science Fund (30671212) are highly appreciated. The authors wish to express their appreciation to Prof. Suichu Qin and Zhangquan Shen, for their valuable suggestions on the study and to Dr. Xiuying Zhang for her help with English language writing.

\section{REFERENCES}

A. Leduc, P. Drapeau, Y. Bergeron, et al. Study of spatial components of forest cover using partial Mantel tests and path analysis, Journal of Vegetation Science, 1992, 3: 69-78

B. Hoersch, G. Braunb, U. Schmidt. Relation between landform and vegetation in alpine regions of Wallis, Switzerland. A multiscale remote sensing and GIS approach, Computers. Environment and Urban Systems, 2002, 26: 113-139 
F.W. Davis, S. Goetz. Modelling vegetation pattern using digital terrain data, Landscape Ecology, 1990, 4: 69-80

$\mathrm{Fu}$ YuLu, Zhang XianYang, Huang YueFu. Relationship analysis between production of Chinese Torreya and meteorologic factors, Shanghai Agricultural Science and Technology, 1999 (1): 69-70 (in Chinese)

Han NingLin, Wang Donghui. Cultivation Techniques of Torreya, China Agriculture Press, Beijing, 2006 (in Chinese)

I.D. Moore, R.B. Grayson, A.R. Ladson. Digital terrain modelling: a review of hydrological, geomorphological, and biological applications, Hydrologic Process, 1991, 5: 3-30

L. Claessens, P.H. Verburg, J.M. Schoorl, et al. Contribution of topographically based landslide hazard modelling to the analysis of the spatial distribution and ecology of kauri (Agathis australis), Landscape Ecology, 2006, 21: 63-76

Li Zhangju, Cheng Xiaojian, Dai Wensheng, et al. Origin of Torreya grandis 'Merrillii', Journal of Zhejiang Forestry College, 2005, 22: 443-448 (in Chinese)

Liu Quan, Liu Hai. Studies on Pattern Recognition of Favourable Ecological Regions of Chinese Torreys, Journal of Zhejiang Forestry College, 1993, 10(2): 122-156 (in Chinese)

M.C. Garcia, M.A. Ortiz, J.J. Zamorano, et al. Vegetation and landform relationships at Ajusco volcano Mexico, using a geographic information system (GIS), Forest Ecology and Management, 2007, 239: 1-12.

Meng Hongfei, Jin Guolong, Weng Zhongyuan. Investigation on resource of ancient Torreya grandis trees in Zhuji City, China, Journal of Zhejiang Forestry College , 2003, 20(2): 134136 (in Chinese)

PCI Geomatics. OrthoEngine reference manual, PCI Geomatics, Ontario, 1998

R. Kalliola, K. Syrjänen. To what extent are vegetation types visible in satellite imagery? Annales Botanici Fennici, 1991, 28, 45-57

Ren Qinliang. An investigation of injury of Torreya Grandis seedlings occurring in dry hot season, Subtropical Forest Science and Technology, 1984(2): $52-53$ (in Chinese)

Ren Qinliang. Biologic features of Chinese Torreya (Torreya Grandis), Economic Forest Researches, 1989, 7(2): 56-60 (in Chinese)

S. Tatsuhara, Y. Antatsu. Predicting the spatial distribution of the abundance of Siebold's beech in a montane cool-temperate region based on environmental factors. Journal of Forestry Research, 2007, 12: 442-451

Wu Jungen, Liu Yu. High Yield Technology Question-answering of Chinese Torreya and Hickory, Beijing: China Meteorological Press, 1994 (in Chinese) 